\title{
Statistical analysis of computer-generated thermal images based on overall modeling of line-scanning process
}

\author{
by MILOVANOVIĆ D., MARINČIĆ A., BARBARIĆ Z. and PETROVIĆ G.
}

1 aculty of Electrical Engineering, P.O.Box 816, 11000 Belgrade, Yugoslavia.

\section{Abstract}

Using our previously developed computer model of line-scanner on moving platform, several synthetic thermal images of a terrain background were produced. The geometrical image deformations, spatlal radiance filtration and influence of the model scene parameters on statistical properties of simple terrain image are analyzed. The change of the mean value, standard deviation, histogram and pixel-topixol correlation of computer-generated thermal images with a scanning angle, indicate a possibility for ndapllve reduction of the data redundancy for an efficient digital image transmission.

\section{Introduction}

The thermal infrared image is produced by the distribution of the radiant emittance of a gene transformed through an optomechanical scanning device into a flux incident on the detector. The video signal at the detector output is proportional to the incident flux from the terraln and the atmosphere, within a specified wavelength range.

In reality the generation of an infrared thermal image is a complex process not easily medeled in a general case. An approach to one-dimensional modeling of the spatial and tratlstical properties of background radiance in intermediate infrared spectral region 2-14 $\mu \mathrm{m}$ was presented by Itakura [1]. The natural terrain modeling (based on a simplified heat balance equation for each image pixel) was suggested by Ben-Yosef et al [2]. Statistics of the radiance gvor the infrared image depends on the imaging system as well. A simulation model SENSAT2. (Sensor-Atmosphere-Target) was presented by Richter [3]. This model was developed for remote sensing applications of passive sensors in the infrared spectral region 1-28 $\mu \mathrm{m}$. A mothod for simulating of non-homogeneous sea surface images (based on modeling bottomup processes) obtained by foiward-looking infrared (FLIR) sensors in the 8-12 $\mu \mathrm{m}$ band is presented by Wilf and Manor [4]. A new computer model (based on two-dimensional terrain itatistics and bottom-up process simulation) for the generation of thermal images by lineveanning from low altitude aircraft was developed by Barbaric et al [5], in the spectral range 8$12 \mu \mathrm{m}$.

For visual analysis, thermal images are usually recorded on film or transmitted and displayed on a video monitor. Digital signal processing techniques have important role in lineBcanning systems due to the increasing need to maintain high image quality from sensor to display and efficient interface to real-time data link. Various image processing techniques can be used for sensor artifact suppression and data compression techniques for solving the anormous instantaneous data rates problem [6].

\section{Computer simulation procedures for thermal image generation}

The computer model used in this work has simulated a simplified real system: flat terrain with flat non-overlapping objects, homogeneous atmosphere and the scanning device moving at a constant speed-to-altitude ratio, with the scanning plane perpendicular to the terrain plane. A schematic diagram of simulation procedures is shown in figure 1.

The terrain is simulated as a periodic repetition of the terrain base area, made from ground and isolated square shaped hot plates of different sizes. Normal probability density 
function and exponentially decreasing isotropic autocorrelation function are used to model natural fluctuation of terrain two-dimensional temperature distribution. The objects in the terrain base area are defined by their non-overlapping contours and specified statistical parameters: mean temperature distributions, the standard deviations and the correlation lengths. Desired discrete temperature distribution was achieved by filtering in the two-dimensional discrete Fourier transform domain.

We assumed that the terrain radiates as a black body following Planck's radiation law. The average temperature of the natural terrain is between 250 and $350 \mathrm{~K}$, which means that the peak radiation is in the range from 11.6 to $8.3 \mu \mathrm{m}$, respectively. At the same time this wavelength range is convenient because of the small reflected spectral radiance, high atmospheric spectral transmittance and good spectral response of detectors. The atmosphere attenuation $\tau$ is a function of the scanning angle $\Theta$ and the average transmittance $\tau$ at the nadir $\tau=\tau^{\sec \theta}$.

The opto-mechanical scanner can be interpreted as a device collecting radiance flux from discrete elementary areas within the resolution cell on terrain and the atmosphere volume defined by the solid angle of the sensor cone. The collected radiations act on the detector, which converts it into a signal at the thermal image plane. This system is successfully modeled by the optical transfer function of the sensor and geometrical coordinate transformation from the terrain to the image plane.

The thermal images can be efficiently generated pixel-by-pixel by summation of the discrete series of spatial harmonics of the terrain radiance distribution multiplied by the optical transfer function of the sensor. The voltage at the detector output is obtained in the form of a double sum [5]:

$$
V(i, k)=\sum_{m=0}^{M-1} \sum_{n=0}^{N-1} K_{1}\left[\tau L_{C}^{T}(m, n)+L_{C}^{A}(m, n)\right] H\left(m, n, \theta_{k}\right) \exp \left[2 \pi j\left(\frac{m}{M} i+\frac{n}{N} k\right)\right]
$$

where $K_{1}=A_{0} k_{0} R \alpha_{0} \beta_{0}$ is a constant, $i=0,1,2, \ldots$ and $k=0,1,2, \ldots$. The numbers $\mathrm{M}$ and $\mathrm{N}$ are selected so that all available samples of radiance over the terrain base area are included. The radiances $L_{C}^{T}(m, n)$ and $L_{C}^{A}(m, n)$ are the discrete Fourier transforms of the terrain radiance $L_{C}^{T}(i, k)$, and the atmosphere radiance $L_{C}^{A}(i, k)$. The normalized transfer function of sensor square shaped aperture is $H\left(m, n, \theta_{k}\right)$ where $\theta_{k}=\operatorname{artan}(k \Delta y / H)$ and $\mathrm{H}$ is the altitude of the moving platform. In the calculation of sinc type function $H\left(m, n, \theta_{k}\right)$ Hermitian symmetry with respect to $m$ and $n$ is taken into account.

The output signal from the detector has to be resampled and quantified. We assumed continuous scanning without overlap or underlap of adjacent scanning tracks at the nadir and resolution cells along the scan direction. Line-scanning parameters are: plane components of constant angular instantaneous field of view $\alpha_{0}=\beta_{0}=1.5 \mathrm{mrad}$, step along flight direction at the nadir $\Delta \mathrm{X}=\mathrm{H} \alpha_{0}=1 \mathrm{~m}$ and scanning angular step $\Delta \Theta=\beta_{0}$.

The equations describing the model have been programmed in high computer language FORTRAN. The program input data are the dimension of the terrain base area and the objects contours, statistical parameters for ground and objects areas, atmospheric conditions, flight parameters, sensor characteristics and scanning angle ranges. Computer processing procedure is very time consuming.

Several thermal images of a terrain background with square hot plates were generated by using the developed computer model. The selected image frames correspond to the scanning angle ranges: $0^{\circ}<\Theta<5.5^{\circ}, 15^{\circ}<\Theta<20.5^{\circ}, 30^{\circ}<\Theta<35.5^{\circ}, 40^{\circ}<\Theta<45.5^{\circ}$ and $55^{\circ}<\Theta<60.5^{\circ}$. The dimensions of each frame are $64 \times 64$ pixels. We selected the terrain base area of $64 \times 256$ points as a compromise between computation time and good statistics. Statistical parameters of the terrain are $m_{G}=298 \mathrm{~K}, \sigma_{G}=1.25 \mathrm{~K}$ and the correlation length $d_{G}=2 \mathrm{~m}$. The dimension of 
http://dx.doi.org/10.21611/qirt.1994.003

qquare plates are $16 \mathrm{~m} \times 16 \mathrm{~m}, 8 \mathrm{~m} \times 8 \mathrm{~m}, 4 \mathrm{~m} \times 4 \mathrm{~m}$ and $2 \mathrm{~m} \times 2 \mathrm{~m}$ with statistical parameters $m_{i}, 306 \mathrm{~K} ; \sigma_{P}=1 \mathrm{~K}$ and the correlation length $d_{p}=4 \mathrm{~m}$. A representative example of the three lypes of synthetic frames with various dimensions of square plates corresponding to the nadir $\left(0^{\circ} \circ \Theta<5.5^{\circ}\right.$ and scanning angle range $55^{\circ}<\Theta<60.5^{\circ}$ is shown in figure 2 . The image frames were quantified with $\mathbf{2 5 6}$ gray levels, but in this paper they are printed with limited number of gray levels.

\section{Analysis of generated thermal images}

In our previous works we investigated basic line-scanning effects, atmosphere radiation and attenuation role in the three characteristic cases for different ratios of average aimosphere to ground temperature[5], and isolated influence scene parameters to the statistical properties of thermal images in the two extreme cases for uncorrelated and correlated terrain background [1]: In this paper we are continuing the investigation of isolated line-scanning effects in case of simple objects on correlated terrain temperature distribution and without the influence of atmosphere. We have analyzed geometrical image deformations, spatial radiance filtration due to finite dimension of detetector aperture and image statistical parameters: mean value, standard deviation, histogram, entropy and pixel-to-pixel two-dimensional correlation as a function of the scanning angle and spatial resolution.

\subsection{Geometrical image deformations}

The geometrical effects of line-scanning are clearly seen on representative computergenerated thermal image frames corresponding to the scanning angle range $55^{\circ}<\Theta<60.5^{\circ}$ shown in figure 2. Images, displayed on uniform grid with square shaped pixels, are nonlinearly compressed by artan law due to constant instantaneous angular field of view and increasing area of scanning optics projection (resolution cell) on the terrain at various scanning angles. The area of the resolution cell increased as $\sec ^{3} \theta$ i.e. by $\sec ^{2} \theta$ in the along scan direction and $\sec \theta$ in the along flight direction. The use of constant angular sampling rate causes overlapping adjacent scanning tracks, seen as changes in the shape and size, and blurred edges of the plates. In practice, those sensor artifacts must be suppressed by various image processing techniques which include resampling, destripping and interpolation. Also, bofore applying any data compression, it is first necessary to remove all oversampled pixels from the image. Particularly, for transform coding a geometrically correct image should be created [8].

\subsection{Statistical image analysis}

Another effect of the line-scanning is the change of the image gray level fluctuation with the increasing scanning angle due to spatial non-invariant terrain radiance averaging on area of resolution cell i.e. decrease of spatial resolution or equivalently decrease in the bandwidth of scanning optics transfer function. The statistical parameters of selected computer-generated thermal frames are calculated.

Image histograms of representative synthetic thermal frames are shown in figure 3. One can see that a relative frequency occurrence of gray levels i.e. image probability density function is changed as a function of scanning angle but is still of the Gaussian type as we oxpected it due to system linearity. Also, its distribution is related to the line-scanning spatial resolution that approaches the normal distribution owing to the central limit theorem [2], regardless of beginning distribution. Histograms of the frames with objects at nadir have two Gaussian peaks on positions of mean values of background and hot plates. With increasing scanning angle, the plates histogram peaks disappears owing to line-scanning effects described in Section 3.1, and the background histogram peak becomes slightly asymmetric.

The mean value of three types of computer-generated thermal frames decreases as a function of the scanning angle as shown in figure $4 \mathrm{a}$. In figure $4 \mathrm{~b}$. the standard deviation of the three types of synthetic frames gray levels as a function of the scanning angle is shown. The standard deviation also decreases with the scanning angle due to increasing area of the 
resolution cell. The rates of mean value and standard deviation decay depend on the terrain type i.e. total area of plates, with expected behavior that for the scanning angles near to the horizon statistical values of all frames have the same value. Changes of mean value and standard deviation with the scanning angle can be used in selecting an optimal quantification of thermal image signal samples as a function of scanning angle.

Figure 4c. shows statistical results for the two-dimensional autocorrelation coefficients for neighboring pixels. In the along flight direction the autocorrelation coefficient $\left(R_{1}^{1}\right)$ increases with the scanning angle because in this case we have increased overlapping of the acjacent scanning terrain tracks. The along scan direction correlation coefficient $\left(R_{2}^{l}\right)$ decreases. It should be noticed that in spite of the assumption that the terrain is assumed isotropic, the correlation of the thermal iniage formed by the line-scanning is anisotropic. The local high correlation of frames can be used in selecting the weighting coefficients in the adaptive predictive coding and in analyzing the spatial frequency content of an image for transform coding techniques [8].

Figure $4 d$. shows the decay of first-order entropy of the three types computer-genirated thermal image as a function of scanning angle. The entropy is calculated from histograms as the average value of the self-information of the selected image frames. The change of eintropy with the scanning angle proves that variable word length coding can be used. The optimum entropy coding depends on image statistics and therefore must be optimized for some ypical class of terrain.

\section{Conclusion}

Based on the analysis of statistical parameters of computer-generated thermal images of simplified terrain, we expect that the changes of parameters that were found can be advantageously used for the redundancy reduction and the selection of predictive, transform and entropy adaptive coding. The line-scanning signal is a non-standard one as compared with the standard composite television signal therefore the redundancy reduction techniques used in a television are not directly applicable to a line-scanning thermal image. In practice, image quality criteria for infrared line-scanned image differ from those used for television signal. And finally, the efficiency of the proposed techniques for data compression deperids on the type of terrain and requires further study in order to arrive at an optimal solution.

\section{REFERENCES}

[1] ITAKURA, (K.) TSUTSUMI (S.) and TAKAGi (T.) - Statisticai properties of the background noise for the atmospheric windows in the intermediate infrared region. Infrared Physics Vol.14, pp.17-29, 1974.

[2] BEN-YOSEF (N.), WILNER (K.) and ABITBOL (M.) - Measurement and modeling of natural desert terrain in the infrared. Optical Engineering, Vol.27 No.11. pp.928-932, 1988.

[3] RICHTER.(R.) - Infrared Simulation Model SENSAT-2. DFVLR-FB 87-10, Institut fur Optoelektronik, Oberpfaffenhofen, 1987.

[4] WILF (I.) and MANOR. (Y.) - Simulation of sea surface images in the infrared. Applied Optics, Vol.23 No.18, pp.3174-3180, 1984.

[5] BARBARIC (Z.), MARINCIC (A.) , PETROVIC (G.) and MILOVANOVIC (D.) - Thermalimage generation by line-scanning technique: $A$ new computer model. Applied Optics Information Processing, Vol.33 No.14, pp.2883-2890, 1994.

[6] MCCRACKEN (W.) and CORBETT (W.) - Overview of digital signal processing for line-scan imagery. SPIE Vol. 24 pp.132-149, 1983.

[7] Z. BARBARIC, (A.) MARINCIC, (G.) PETROVIC and MILOVANOVIC (D.) - Thermal images generated by a line-scanning technique: Statistical properties. Applied Optics, accepted for publication, 1994.

[8] LEMNE (G.) and JOHANSSON (B.) Design considerations for high-speed transform image compression. SPIE Vol. 694 pp.55-61, 1986. 
Terrain

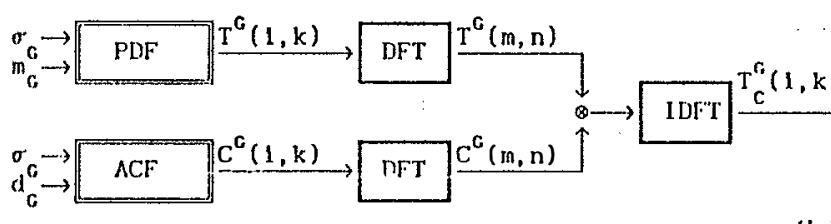<smiles>CCCCC</smiles>
$T_{c}^{T}(1, k)$

$\underset{0}{m_{0}^{0} \rightarrow \mathrm{PDF}} \stackrel{\mathrm{T}^{0}(1, \mathrm{k})}{\mathrm{DFT}} \mathrm{T}^{\mathrm{o}}(\mathrm{m}, \mathrm{n}$ $\underset{0}{d_{0}^{0} \rightarrow A C F} C^{0}(1, k) \longrightarrow D F C C^{0}(m, n)$

Atmosphere

$\underset{m_{A}^{A} \rightarrow P D F}{\sigma_{D} \rightarrow T^{A}(1, k)} \longrightarrow T^{A}(m, n)$

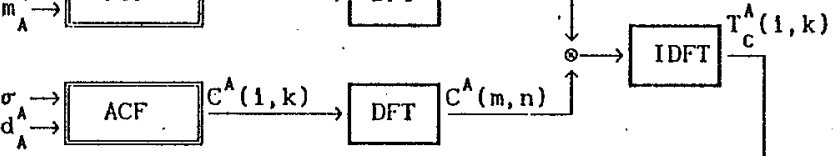

$\bar{\tau} \rightarrow \bar{\tau}^{\sec \theta} \mathrm{\tau}$
$\longrightarrow$

$$
\left.z_{1 t}^{\tau \rightarrow \frac{(1-\tau)\left[Z_{1 t} T^{\wedge}(1, k)-Z_{2 t}\right)}{\downarrow_{L_{C}^{A}(1, k)}}}\right|^{\downarrow} \leftarrow-\tau
$$

Scanning optics

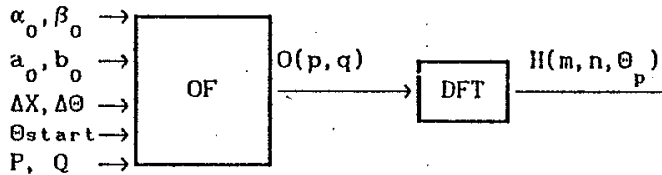

DFT

$$
\begin{aligned}
& \text { PDF - probability density function } \\
& \text { ACF - autocorrelation function } \\
& \text { DFT - discrete Fourler transform } \\
& \text { IDFT - Inverse DFT } \\
& \text { OF - optical function } \\
& \text { II - optical transfer function }
\end{aligned}
$$

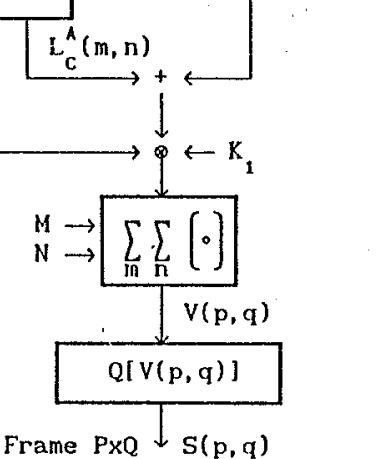

Fig. 1. - Computer simulation procedures for generating an infrared thermal image. 
http://dx.doi.org/10.21611/qirt.1994.003

a)
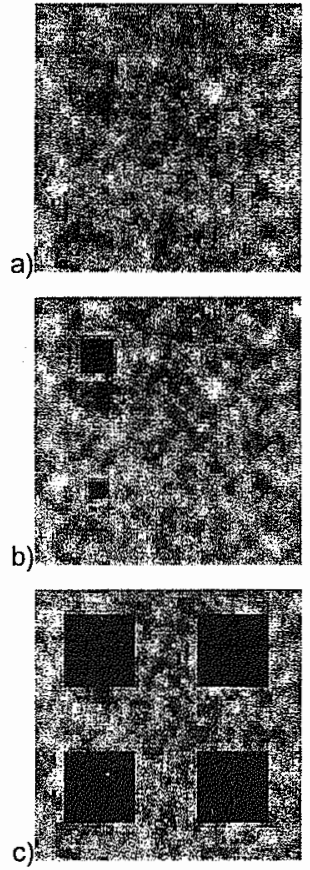

Scan direction $\rightarrow$
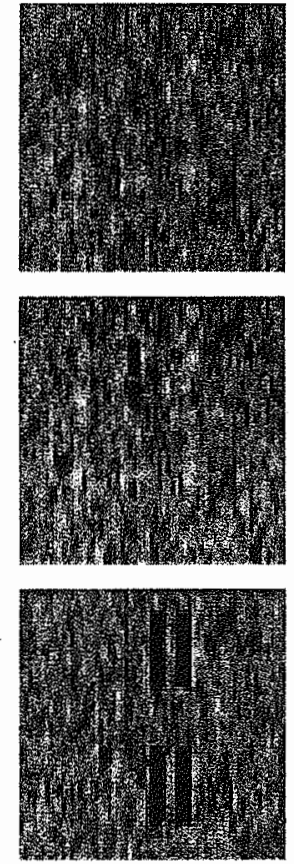

b)
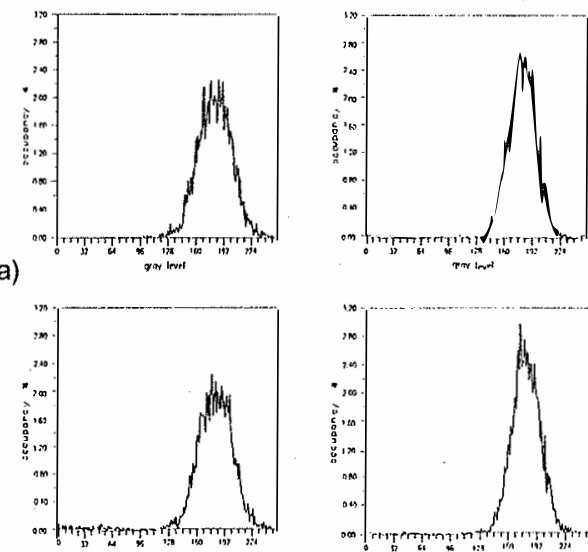

b)

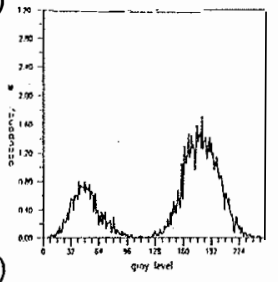

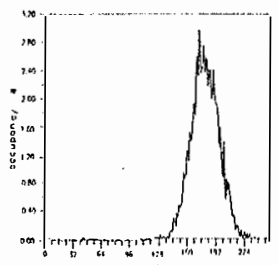

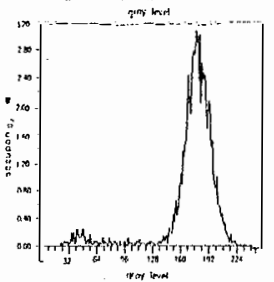

Fig.2. - Representative frames of computergenerated thermal images of three types terrain scanned in the angle ranges $0^{\circ}<\Theta<5.5^{\circ}$ and $55^{\circ}$ $<\Theta<60.5^{\circ}$ : a) The terrain background; b) The four unequally sized $(16 \mathrm{~m} \times 16 \mathrm{~m}, 8 \mathrm{~m} \times 8 \mathrm{~m}, 4 \mathrm{~m} \times 4 \mathrm{~m}$ and $2 \mathrm{~m} \times 2 \mathrm{~m})$ and $\mathrm{c})$ the four equally sized $(16 \mathrm{~m} \times 16 \mathrm{~m})$ hot plates on the background (black is hot).

a)

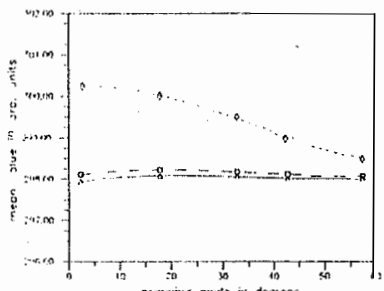

c)

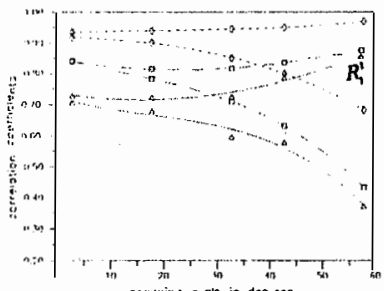

b)
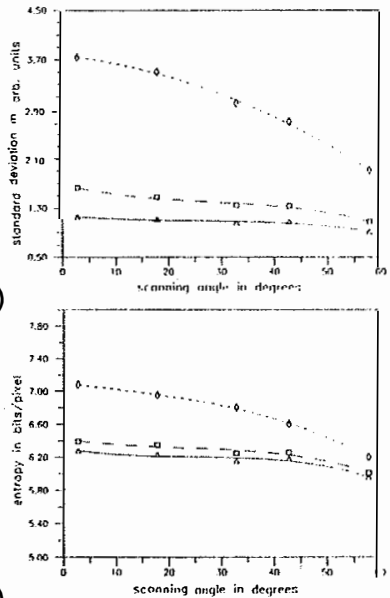

Fig.4.- The variation of statistical parameters of selected computer-generated thermal frames of three types terrain shown in figure $2(-$ figure $2 a,-$ figure $2 b, \ldots .$. figure $2 c)$ as a function of scanning angle: a) mean value, b) standard deviation, c) autocorrelation coefficients ( $R_{1}^{\prime}$ in flight direction and $R_{2}^{\prime}$ in scan direction and d) entropy. 